\title{
De la accesibilidad a la funcionalidad del territorio: una nueva dimensión para entender la estructura urbano-residencial de las áreas metropolitanas de Santiago (Chile) y Barcelona (España) ${ }^{1}$
}

\author{
Jorge Cerda Troncoso² y Carlos Marmolejo Duarte ${ }^{2}$
}

\begin{abstract}
RESUMEN
Uno los factores clave en el desarrollo de los sistemas urbanos discontinuos es la accesibilidad, sin embargo, este se ha limitado a generar medidas basadas en el análisis de tiempo, distancia y coste para acceder desde un punto al resto del sistema urbano. La accesibilidad no solo está determinada por las redes de transporte, sino, sobre todo, por la movilidad, reflejo físico de las voluntades de las personas para moverse a través de la ciudad. En esta investigación se propone un enfoque basado en la movilidad; se trata de un indicador (funcionalidad urbana) superador del enfoque físico y más próximo a su naturaleza social. Una vez construido, tanto para Barcelona como para Santiago, se analiza, mediante un modelo econométrico, su eficiencia para explicar la distribución de la población. Los resultados sugieren que la funcionalidad tiene una capacidad explicativa superior a las medidas tradicionales. Asimismo, la elasticidad de la demanda para realizar viajes comerciales más largos es superior a la de los laborales, más rígida. En Barcelona la demanda tiene mayor rigidez ante el incremento de la distancia respecto de Santiago.
\end{abstract}

Palabras clave: Funcionalidad, accesibilidad, movilidad, densidad residencial.

\begin{abstract}
One of the most important elements in the development of discontinuous urban systems is accessibility; nonetheless, this concept has been limited to indicator based on time, distance and cost in order to go from one point to another inside the urban system. Accessibility is not only determined by transport networks, but mainly by mobility, a physical reflection of peoples will to move across city. This investigation proposes an alternative way to compute accessibility, called urban functionality. This indicator is based on mobility analysis, and so on the social nature of accessibility. To prove the efficiency of urban functionality to explain residential distribution, two metropolitan areas were studied: Barcelona and Santiago de Chile. The results suggest that urban functionality has a better capacity to explain than traditional methods based on time and distance. In addition, it can be observed that non mandatory mobility has a more rigid demand if it's compared to obligatory mobility (i.e. travel-to-work). Barcelona is more rigid on mobility if is compared with Santiago.
\end{abstract}

Key words: Functionality, accessibility, mobility, residential density.

1 Artículo recibido el 24 de noviembre de 2009 y aceptado el 15 de enero de 2010.

2 Centro de Política de Suelo y Valoraciones, Universidad Politécnica de Cataluña (España). E-mail: jorge. francisco.cerda@upc.edu; carlos.marmolejo@upc.edu
El primer autor contó con el apoyo del Comisionado para Universidades e Investigación del Departamento de Innovación, Universidades y Empresa de la Generalidad de Cataluña y del Fondo Social Europeo. 
Hace tiempo que el crecimiento periférico de los sistemas metropolitanos ha dejado de ser denso, diverso y continuo. Los trabajos de Berry (1980), Hall \& Hay (1980), y Van der Berg (1982) han documentado el acusado proceso de suburbanización que, desde la década de los años 60 y 70, ha caracterizado a las metrópolis norteamericanas y de Europa septentrional, y más recientemente a las ciudades históricamente compactas de la Europa meridional (Nel·lo, 2002). De esta manera, el crecimiento metropolitano de centralización absoluta y relativa, en tejidos compactos, ha dado paso a otro de descentralización relativa e incluso absoluta, en tejidos poco densos.

En América Latina el modelo de crecimiento por dispersión también existe, aunque es más reciente en relación a otras geografías, y en esa medida, es menos intenso y aún coexiste con el modelo de "mancha de aceite" de baja densidad (Hoyos, 2000; Ortiz y Morales, 2002; Aguilar, 2002). Naturalmente, los crecimientos suburbanos en las regiones metropolitanas latinoamericanas tienen sus especificidades propias, dadas por la gran desigualdad en el reparto de la riqueza y el bajo nivel de dotación infraestructural. Así, en un extremo, se tiene a las ciudades cerradas para grupos de renta media y alta, como lo han documentado en Argentina (Thuillier, 2005; Vidal-Koppmann, 2008), en México (Rodríguez, 2006; Enríquez, 2007) o bajo la acepción de megaciudades valladas o privatópolis en Santiago de Chile (Hidalgo et al., 2008).

Lo relevante de la cuestión es por qué los nuevos crecimientos han adoptado una fisonomía diferente a la que tradicionalmente había dominado en el desarrollo por continuidad. En este sentido, la explicación de la dispersión urbana ha tenido un fuerte enfoque físico (Galster et al., 2000), y en menor grado económico (Rodríguez-Bachiller, 1986; Caruso et al., 2008). Los aspectos normativos (zonificaciones que restringen el desarrollo) y geográfico-físicos claramente promueven la discontinuidad en los asentamientos y en la expansión urbana. Por lo anterior, lo relevante es determinar las condiciones que permiten desarrollar territorios alejados del centro urbano, haciéndolos parte de la expansión metropolitana. Dicha condición debe ser general para el fenómeno urbano en su con- junto, y no solo circunscribirse a las áreas de expansión, como es común en estudios de este tipo.

Sin lugar a duda una de las precondiciones que un sitio debe tener para que se desarrolle urbanísticamente es la accesibilidad. Sin embargo, en la literatura, este concepto ha tenido una evolución desde el enfoque de mensurar el nivel de proximidad de un sitio en relación con los otros que conforman el sistema urbano, hasta una connotación social integral del beneficio, aptitudes, y evolución social de los individuos. Las primeras aproximaciones han intentado medir el nivel de dificultad para superar el espacio (por ejemplo ponderando velocidades, el número de arcos de la topología de las redes que se han de superar, los costes, entre otros). Frente a la crítica relativa a que la accesibilidad no debería ser solo un indicador físico de la impedancia del espacio, sino, sobre todo, social, es que ha evolucionado el concepto hacia "la característica que permite a los individuos ejercer el derecho a la ciudad" (Miralles, 2003: 14). En el contexto de esta última aproximación, la accesibilidad debería contemplar la forma en cómo la ciudadanía está dispuesta a viajar a lo largo del espacio para satisfacer sus necesidades. No basta, por tanto, con tener una buena conexión, si no se tiene claro hacia dónde se realizan los viajes ni las condiciones particulares de los viajeros.

Además de la ambigüedad antes descrita, la relación entre accesibilidad y la distribución espacial de las actividades es también un campo complejo y contradictorio. Así lo muestran algunos autores (Oyon, 1999; García, 2008; Flichy, 2004), al constatar la existencia de estudios que, con distintas fuentes informativas, demuestran que la dispersión urbana, por una parte, reduce la longitud media de los viajes $y$, por otra, aumenta dicha longitud. O también respecto de cómo la congestión de los centros urbanos induce el surgimiento de subcentros periféricos.

En este contexto, la presente investigación primero plantea una nueva dimensión de acceso, denominada funcionalidad territorial, la cual incorpora el comportamiento real de la movilidad de la población, bajo el enfoque de cómo los habitantes utilizan la ciudad para satisfacer sus necesidades. Posteriormen- 
te esta nueva dimensión se compara con las medidas tradicionales de acceso (distancia, tiempo), en su capacidad para explicar la estructura de densidades residenciales. El desafío de la investigación es detectar comportamientos y parámetros sociales (reales) de movilidad, que sustituyan los comportamientos teóricos que actualmente utilizan los distintos modelos de transporte, y que tenga una validez explicativa de patrones locacionales residenciales.

El documento se ha estructurado de manera que el primer y segundo apartado presentan un desarrollo conceptual que construye el enfoque de funcionalidad territorial sobre el cual se basa la investigación. A continuación se presentan tres apartados con los objetivos, ámbito de estudio y metodología desarrollada, para finalmente en los dos últimos apartados presentar los resultados obtenidos y las conclusiones finales de la investigación.

\section{Discusión conceptual}

La relación entre transporte y estructura urbana ha estado tradicionalmente basada en un paradigma del tipo causal, es decir, una predeterminación de un factor a partir del otro. Los modelos de transporte y usos de suelo (LUT) trabajan con dicho paradigma en forma explícita (de forma estática y dinámica).

La consecuencia que genera el paradigma de la causalidad, ya sea en un sentido o en otro, lleva a lo que Miralles (2002) denomina la urbanística o a la transportística; la primera se ocupa de estudiar cómo la estructura física de la ciudad produce la demanda de movilidad, y la segunda cómo la oferta de transporte induce la localización de actividades.

El elemento que tradicionalmente se ha considerado como articulador entre el transporte y la estructura urbana es la accesibilidad. Pero este término tiene varias acepciones, que han ido cambiando a lo largo del tiempo, tanto a nivel conceptual como metodológico.

En la línea conceptual, Miralles y Cebollada (2003) entienden que la accesibilidad contempla una dimensión asociada a la fric- ción espacial producto de la separación entre actividades e individuos (acceso), pero también considera el número de opciones reales (factibles desde el punto de vista económico y cultural, entre otros) que tienen los ciudadanos para desarrollar las actividades. La concepción física se asocia a una situación netamente de localización espacial, referida a la contigüidad espacial en fenómenos de difusión de actividades, innovaciones y externalidades (Batty, 2003).

La visión económica (Martínez, 1994) supone que los individuos (agrupados en hogares) obtienen beneficios del ejercicio de variadas actividades, cuyo total intentan maximizar mediante un uso óptimo de sus recursos tiempo e ingreso (esto constituye la ley de comportamiento del individuo racional). La firma, por su parte, cualquiera sea su actividad, pretende maximizar sus utilidades económicas restringidas por la tecnología e insumos disponibles. Por lo tanto, es la función de utilidad de hogares y la función de producción de las firmas la que establece el valor económico asociado al acceso a cada actividad urbana, y es en virtud de ese valor que se tomarán decisiones de localización espacial. Por lo tanto, para entender el rol del acceso en las decisiones de localización urbana es necesario entender primero las necesidades de interacción con otras actividades que los hogares y firmas tienen implícitas en sus funciones de utilidad y producción, respectivamente, y luego intentar representar tales necesidades y percepciones en medidas apropiadas de acceso. Así, se entiende por accesibilidad a la utilidad o beneficio económico que reporta al hogar o firma el visitar actividades propias de su quehacer, y por atractividad al beneficio económico que extrae el hogar o firma por las visitas que recibe en su entorno. Notar que accesibilidad es un beneficio directamente percibido por los que hacen viajes, mientras que atractividad es un beneficio a las actividades (hogares y firmas) localizadas en los destinos de los viajes.

La visión social de la accesibilidad se encuentra actualmente bajo el concepto de motilidad (Kaufmann, 2004), que se define como la capacidad de entidades (por ejemplo bienes, información e individuos) de moverse en el espacio geográfico y en el espacio social (movilidad social), es decir, la forma 
en que los individuos acceden y se apropian de capacidades para moverse en el espacio físico y en su estatus social (espacio social). Así, el acceso es parte de la motilidad, y tiene que ver con la gama de posibles movimientos en relación al espacio, tiempo y otras condiciones contextuales del territorio (actividades, población e infraestructura, entre otros). Al acceso se le suman, primero las competencias de los individuos (habilidades y capacidades), y finalmente la apropiación que tiene que ver con cómo los individuos interpretan y toman sus decisiones en función de las condiciones de acceso y competencias.

En cualquiera de estas concepciones (física, económica, y social), la accesibilidad es un concepto no único, pero sí condicionado por los componentes espaciales, temporales, y por el sistema de transporte como el elemento tecnológico (Miralles, 2002).

A nivel metodológico (métodos de medición), se ha pasado desde la formulación topológica de accesibilidad de la teoría de redes (que además diferencia el concepto de accesibilidad del de conectividad), a la concepción de accesibilidad en los modelos de transporte y usos de suelo (Martínez, 1994; Lautso, 2005), que se asocia a beneficios microeconómicos de los usuarios y/o de la localización (modelos de utilidad aleatoria), o a comportamientos gravitatorios en las interacciones (modelos gravitacionales). Finalmente en la modelación por autómatas celulares (CA), y por modelos basados en agentes (ABM), la accesibilidad se asocia al término de contigüidad o vecindad, cuyo carácter es local y acotado (predefinido), con un claro enfoque proveniente de la física de fluidos y gases, en donde la difusión o dispersión es preferentemente hacia zonas contiguas (Batty, 2003).

Los enfoques expuestos muestran una alta divergencia en concepciones y métodos, donde estos últimos además están altamente determinados por decisiones teóricas que rigen sus cálculos (por ejemplo la función de la fricción de la distancia, como inverso de la distancia al cuadrado, funciones exponenciales o lineales, entre otros).

La causalidad en la relación entre transporte y ciudad, junto a la ambigüedad del concepto de accesibilidad, han dado pie a una serie de críticas, surgidas principalmente desde los enfoques filosóficos, sociológicos y geográficos, preocupados por la relación entre la tecnología y los comportamientos sociales, específicamente en lo referente a la dimensión espacial de las estructuras sociales.

A pesar de estas reflexiones, no se ha logrado modificar el paradigma dominante (causalidad) de las metodologías de los estudios de transporte urbano (modelo clásico de transporte de cuatro etapas, y su evolución a los modelos LUTM o 5 etapas). Dicha metodología ha gozado, además, del reconocimiento político y de los planificadores por ser eficaz, científica y clara en la evaluación de planes y proyectos. A pesar de que esto es efectivamente así, no deja de ser un enfoque parcial y pragmático, orientado generalmente a resolver saturaciones de la capacidad de transporte para satisfacer las necesidades de interacción de las áreas de expansión, dejando de lado la discusión conceptual que se da entre la movilidad, la localización de actividades y las funciones urbanas (Miralles, 2002).

Por otra parte, al analizar las razones que subyacen en la expansión de las metrópolis desde la perspectiva de la estructura de actividades en el espacio, surgen tres conceptos que coexisten: centralidad, función y funcionalidad.

La acepción más simple de centralidad hace referencia al punto que, dada su posición geométrica en el espacio geográfico, o su dotación de infraestructuras de comunicación, es capaz de minimizar el esfuerzo para llegar a él desde el resto del territorio, y en ese sentido, es susceptible de centralizar funciones (es un buen punto de distribución de servicios, por ejemplo).

Por otra parte, la función puede definirse como: el conjunto actividades que se influyen entre sí regularmente por medio de relaciones que hacen posible que el sistema social continúe operando. Función es, por tanto, el equivalente de actividad. Las actividades pueden ser de tipo administrativo, comercial, industrial, turístico, de manera que cada centralidad (punto de buena conexión) puede concentrar una o varias actividades. 
Desde las primeras aportaciones de Christaller se sabe que el área de influencia de las centralidades depende de la especialización de las funciones y además, en el caso de la distribución de bienes y servicios, de la elasticidad en su consumo. De tal forma que los centros con funciones de demanda inelástica ante la variación del precio tienden a devenir en lugares centrales y a tener áreas de influencia amplias (hinterland); mientras que los centros con funciones de demanda elástica tienden a localizarse en los subcentros.

Un concepto semánticamente cercano a función, pero claramente distinto es el que se refiere a la funcionalidad urbana. En términos simples, la funcionalidad urbana es la interacción que se genera entre las partes de la ciudad, para satisfacer las necesidades de los residentes y de las actividades localizadas. Así, la estructura urbana está compuesta, al menos, por funciones (nodos) y funcionalidades (relaciones). La funcionalidad o interacción suele materializarse a través de flujos de materia, energía, información y personas entre los nodos (funciones). Dicho de otro modo, la funcionalidad da cuenta de cómo funciona la ciudad en el contexto de relaciones espacialmente localizadas, tomando en consideración, a diferencia de las aproximaciones tradicionales del concepto de accesibilidad, la forma en cómo la población está dispuesta a emplear su tiempo de viaje, elegir sus destinos y rutas.

Como se dijo antes, los nodos más centrales suelen concentrar funciones, que unidas al nivel de acceso (facilidad de paso o de viaje) de la red de interacción, genera jerarquías o niveles distintos de funcionalidad para distintos territorios (posibilidad de funcionar).

\section{El análisis de la estructura urbana desde la perspectiva de la funcionalidad}

Cerda (2008) plantea el concepto de cuenca operacional o cuenca funcional de una ciudad para referirse al espacio en el cual se puede considerar que la ciudad tiene una influencia funcional, es decir, que atrae interacciones (en un número significativo). Siguiendo a dicho autor, el funcionamiento de una ciudad tiene directa relación con el comportamiento de los usuarios de la misma, ya que son ellos con sus características y decisiones los que actúan, materializando la interacción. Por lo que cabe indagar el comportamiento de un residente tipo, en la realización de interacciones al interior de la ciudad, con propósitos específicos, y a su vez, cómo este proceso influye en el comportamiento locacional de las actividades en la ciudad.

La hipótesis de partida de la presente investigación plantea que el potencial de urbanización de un territorio (preferentemente en la función residencial) depende del nivel de funcionalidad urbana del mismo. Dicho de otro modo, depende de la probabilidad de acceder, desde ese territorio, a las distintas centralidades funcionales (actividades) que presenta la ciudad y que proveen de servicios y productos a la población de dicho territorio. A esto se ha denominado probabilidad funcional de un territorio. De manera que cuanto más grande sea dicha probabilidad funcional, ya sea por su proximidad a los puntos de provisión de servicios y empleo (nodos centrales), o por su acceso real (nivel de servicio de las redes condicionado por el comportamiento de los usuarios), mayor es la probabilidad que se urbanice; y además que lo haga de manera densa. Dicho potencial de urbanización se hará realidad dependiendo de una serie de factores, distintos al funcional, que podrían clasificarse en tres grupos: 1) decisiones administrativas relacionadas con el destino del suelo (por ejemplo: áreas protegidas, agrícolas, de riesgo ambiental); 2) decisiones individuales relacionadas con la retención del suelo a la espera de su futura revalorización; 3) problemas relacionados con la gestión de suelo, como los concernientes a la situación jurídica de los derechos sobre ellos obrantes, o bien con la tramitación administrativa de los instrumentos necesarios para su desarrollo (planes y permisos, entre otros).

La principal novedad de esta aproximación de análisis de la ciudad, es que la probabilidad funcional internaliza el comportamiento de los individuos, es decir, su disposición a viajar en función de la utilidad o conveniencia que un viaje le significa, dada la utilidad que le reporta el destino (propó- 
sito de usos de la ciudad), condicionado por el medio (de transporte) en el que viajan, y sus posibilidades reales para viajar de la manera que desean. Se trata, por tanto, de un enfoque de la accesibilidad eminentemente social.

\section{Objetivos y ámbitos del estudio}

El objetivo empírico de esta investigación es cuantificar y caracterizar la funcionalidad urbana, en su dimensión de movilidad ${ }^{3}$, para posteriormente determinar su efecto (comparativamente con otras medidas de acceso más tradicionales) sobre la estructura locacional de la función residencial (densidad de habitantes).

Con el objeto de probar la hipótesis se eligieron dos contextos urbanos diferentes, tanto en su nivel de desarrollo como en su estructura urbana. Así, el ámbito espacial y temporal del estudio corresponde al Área Metropolitana de Santiago de Chile (años 1991 y 2001), y a la Región Metropolitana de BarceIona (año 2001), considerando como unidad de análisis las zonas de transporte utilizadas por las encuestas de viajes correspondientes.

La información utilizada para desarrollar la investigación proviene, básicamente, de las encuestas de movilidad cotidiana. Así, el origen de la información de viajes para ambas ciudades fue, para Santiago, las Encuestas Origen Destino (EOD) desarrolladas por la Secretaría de Transporte (SECTRA) del Ministerio de Planificación, para los años 1991 (517 zonas) y 2001 (715 zonas). Para Barcelona, la Encuesta de Movilidad Cotidiana (EMQ), desarrollada por la Autoridad del Transporte Metropolitano (ATM), de la Generalitat de Cataluña, para el año 2001 (582 zonas). De manera complementaria se utilizó el número de locales no residenciales clasificados por su destino (por ejemplo comercial) proveniente de los censos de locales. Ambas fuentes de datos permiten caracterizar tanto la estructura urbana como la funcionalidad del territorio,

\footnotetext{
3 Ya que existe otra dimensión que está siendo desarrollada por los autores, como es el tiempo de estadía en cada actividad.
}

incluso distinguiendo propósitos de viaje, a escala intramunicipal (zonas de transporte).

\section{Presentación sintética de las áreas metropolitanas}

La ciudad de Santiago de Chile se enmarca dentro de la denominada Región Metropolitana, que está compuesta por 52 municipios, distribuidos en seis provincias. La ciudad de Santiago, usualmente conocida como el Gran Santiago, se refiere al área continua conformada por 34 municipios (comunas) de la región, los cuales fueron agrupados por el primer instrumento de normativa territorial intercomunal el año 1997 (PRMS). En el año 2002, la población de la Región Metropolitana era de 6.061.185 habitantes, correspondiendo al 40,1\% del total país. De esta población, un $90 \%$ se localiza en el Gran Santiago.

Por otra parte, la ciudad de Barcelona se enmarca dentro de la denominada Región Metropolitana de Barcelona (RMB), que está conformada por 164 municipios, dentro de los cuales el Área Metropolitana de Barcelona (AMB) está compuesta por 36 municipios, los que surgen de la unión de las áreas de gestión de tres organismos que son: la Mancomunidad de Municipios de Barcelona (que integra 31 municipios en la gestión de áreas comunes que afectan a: espacios públicos, infraestructuras y vialidad, equipamientos, urbanismo y vivienda), la Entidad del Medio Ambiente (que integra 33 municipios en la gestión de los servicios hidráulicos y el tratamiento de residuos) y la Entidad del Transporte (que integra 18 municipios en la gestión de los servicios de transporte público).

El Cuadro $N^{0} 1$ muestra la evolución demográfica y del suelo urbanizado del Gran Santiago y de la Región Metropolitana de Barcelona.

Respecto de la población urbana (Cuadro $N^{\circ} 1$ ), Santiago presenta un crecimiento, en el último período intercensal, de un 14,8\%, en relación al crecimiento que presenta la Región Metropolitana de Barcelona, que alcanza un $1,7 \%$. La superficie urbanizada de Barcelona, en el último periodo intercensal, experimenta un aumento del $4,3 \%$, mientras que el aumento de Santiago alcanzó un 30,2\%. 
Cuadro $\mathrm{N}^{\circ} 1$

Evolución histórica de la población, extensión urbana y densidad

\begin{tabular}{|c|c|c|c|c|c|c|}
\hline \multirow[b]{2}{*}{ Año } & \multicolumn{2}{|c|}{ Población urbana } & \multicolumn{2}{|c|}{ Área urbanizada (ha) } & \multicolumn{2}{|c|}{ Densidad (hab/ha) } \\
\hline & $\begin{array}{c}\text { Gran } \\
\text { Santiago }\end{array}$ & $\begin{array}{c}\text { RM } \\
\text { Barcelona }\end{array}$ & $\begin{array}{c}\text { Gran } \\
\text { Santiago }\end{array}$ & $\begin{array}{c}\text { RM } \\
\text { Barcelona }\end{array}$ & $\begin{array}{c}\text { Gran } \\
\text { Santiago }\end{array}$ & $\begin{array}{c}\text { RM } \\
\text { Barcelona }\end{array}$ \\
\hline 1940 & 982.893 & 1.681 .826 & 11.017 & & 89 & \\
\hline 1952 & 1.436 .870 & 1.966 .291 & 15.351 & & 94 & \\
\hline 1960 & 1.996 .142 & 2.566 .733 & 21.165 & & 94 & \\
\hline 1970 & 2.820 .936 & 3.579 .072 & 31.841 & & 89 & \\
\hline 1982 / 1981 & 3.902 .356 & 4.234 .725 & 42.080 & & 93 & \\
\hline 1992 / 1991 & 4.754 .901 & 4.299 .790 & 49.270 & 48.951 & 97 & 88 \\
\hline 2002 / 2001 & 5.456 .326 & 4.372 .980 & 64.140 & 51.044 & 85 & 86 \\
\hline $2007^{*}$ & 5.898 .954 & 4.856 .579 & & & & \\
\hline Var 92-02 (\%) & 14,8 & 1,7 & 30,2 & 4,3 & $-11,9$ & $-2,5$ \\
\hline
\end{tabular}

* Proyección en base a censos.

Fuente: Galetovic, 2006; INE (Santiago y Barcelona); CORINE LandCover.

Finalmente, el comportamiento de la densidad es similar, pero de distinta intensidad, ya que en Santiago disminuye un $11 \%$, mientras que en Barcelona disminuye un 2,5\%, en el último periodo intercensal.

El análisis integrado denota que Santiago disminuye su densidad a costa de un crecimiento en superficie mucho mayor que el crecimiento demográfico, lo que ocurre en mucho menor grado en Barcelona. En síntesis, son ciudades morfológicamente distintas.

\section{Metodología}

La metodología desarrollada en este trabajo se basa en cinco etapas, a saber: 1) recopilación y estandarización de la información, 2) cuantificación de la funcionalidad urbana, 3) identificación de la estructura de densidad poblacional urbana, 4) cálculo de la probabilidad funcional territorial, 5) evaluación comparativa de la funcionalidad como variable explicativa de la estructura espacial de densidad poblacional.

Para las ciudades analizadas, se consideran los propósitos de viaje de trabajo, estudio y compras.
Los métodos específicos de cálculo desarrollados en el estudio se explican a continuación.

\section{Patrón de movilidad}

El patrón de movilidad de los individuos en relación a una determinada actividad o propósito $k$ (trabajo, servicios sanitarios, educación, compras, entre otros), es la distribución de probabilidad de tiempos de viaje $(t)$, para satisfacer la actividad $k$, en el sistema urbano. Para construir la distribución de probabilidad de tiempos, se suman todos los viajes con propósito $k$ (con independencia de su origen $i$ y destino $j$ ) que tardan un determinado tiempo $t$ en realizarse, y luego se dividen entre el total de viajes (del propósito $k$ ). Esto genera, por ejemplo, la proporción de todos los viajes con propósito compra, que tardan 10 minutos en realizarse.

Así, $P^{k}{ }_{t}$ es la probabilidad de encontrar, entre todos los desplazamientos observados con propósito $k$, aquellos que se realizan en un tiempo $t$. Cuando la probabilidad se calcula para todos los periodos temporales $t$ (intervalos de 5 minutos), se obtiene el patrón de movilidad de los viajeros para el propósito $k$. 
En concreto, el patrón de movilidad es la distribución de probabilidad simple del tiempo de viaje, en donde se indica la probabilidad que un viaje genérico (independiente de su origen y destino) de propósito $k$, demore un tiempo $t$ determinado.

En los distintos trabajos relacionados con este tema, tradicionalmente se ha utilizado el tiempo medio de viaje (Van Wee et al., 2006), pero como se aprecia, el tiempo de viaje es una variable aleatoria de la movilidad, cuya distribución estadística es en general no simétrica (como se verá más adelante), por lo que no sería correcto utilizar el tiempo medio como valor de referencia.

\section{Probabilidad funcional}

La probabilidad funcional, o dicho de otra forma, la probabilidad de funcionar en la ciudad, también distingue el propósito de viaje (propósito de uso de la ciudad). Este indicador propuesto es puramente empírico. Para su cálculo se acumulan los valores del patrón de movilidad, en forma inversa (partiendo del tiempo mayor al menor). La probabilidad acumulada así construida es la probabilidad funcional para un propósito determinado, y representa la probabilidad de que un viaje, de propósito $k$, dure cuando menos un tiempo $t$. El resultado es una tabla de valores, en donde para cada tiempo se indica el valor de la probabilidad funcional (entre 0 y 1 ) para cada propósito.

En concreto, la nueva medida de accesibilidad, asociada a un determinado tiempo de viaje y para un propósito específico, es el percentil (inverso) en que se ubica dicho tiempo en la curva de probabilidad funcional construida para el propósito de viaje. Para mayor claridad, en la Figura $N^{0} 1$ se aprecia tanto el patrón de movilidad (probabilidad simple), como la probabilidad funcional (probabilidad acumulada).

\section{Cuantificación de la estructura urbana}

La probabilidad funcional de un territorio tiene relación con el propósito de viaje $k$ que se analice. Por este motivo es necesario identificar distintas centralidades de funciones (actividades), asociadas a los distintos propósitos de viaje que se quieren analizar.
La determinación de centralidades o subcentros urbanos es un tema que, a pesar de haber sido muy desarrollado, aún no presenta acuerdo ni en metodologías ni en criterios (Roca et al., 2009). Lo que sí es claro es que es la densidad (empleos, viajes, entre otros) el indicador más ampliamente usado en la literatura que trata el tema (McMillen y McDonald, 1997). Dado que esta discusión no es lo medular en este trabajo, se decidió trabajar con dos tipos de centralidades, donde la primera tiene que ver con la densidad de viajes atraídos por zona y por propósito (viajes totales atraídos dividido por la superficie artificializada de la zona en hectáreas), y la segunda considera la densidad de locales (asociados a los propósitos de viajes). Con esta diferenciación se analizarán los distintos efectos de centralidades funcionales (viajes que Ilegan) y de centralidades de stock (locales de actividad económica).

Para la detección de subcentros en la estructura de densidades antes calculadas, se analizó su distribución estadística para detectar aquellas zonas cuyo valor estuviera por sobre el percentil $75^{4}$, es decir, una zona perteneciente al $25 \%$ de los valores (densidades) más altos. Nótese que para cada propósito de viaje habrá una centralidad diferente, y que un punto en el territorio puede fungir como centralidad para diferentes propósitos.

\section{Cálculo de medidas de acceso y probabilidad funcional de un territorio específico}

La probabilidad funcional antes explicada es un comportamiento de disposición a viajar por un propósito determinado, que es independiente del origen y/o destino del viaje. Ahora, la probabilidad funcional de un territorio está determinada por la capacidad de interacción funcional que tiene este, respecto de distintas centralidades a ser consideradas. Por lo que, una vez determinadas las centralidades, se calcula para cada zona de análisis la distancia (por red de interacción) y

\footnotetext{
4 Este criterio difiere del tradicionalmente usado en estudios de identificación de subcentros, en los que se utiliza que el valor esté sobre una desviación estándar de los residuos generados por un modelo econométrico.
} 
el tiempo ${ }^{5}$ a la centralidad más cercana (para cada propósito). Posteriormente se determina la probabilidad funcional que le corresponde al tiempo de viaje calculado (percentil inverso), a partir de la tabla empírica construida. De esta manera, cada zona queda finalmente caracterizada en función de la distancia, el tiempo y la probabilidad funcional (asociada al tiempo) a cada tipo de centralidad más cercana (densidad de atracción de viajes y densidad de locales), para cada propósito $k$ considerado (trabajo, estudios, y compras).

5 Declarado por los operadores en el caso de Barcelona, y el tiempo medio calculado de las encuestas en el caso de Chile.
Además, con las distintas medidas de accesibilidad calculadas por propósito, se construye una distancia, un tiempo y una probabilidad funcional integrada (que considera todos los propósitos analizados). Para esto se pondera cada medida de accesibilidad por propósito, por la proporción que representan los viajes de propósito $k$, en el conjunto de viajes de la ciudad.

En síntesis, se han construido para cada zona de estudio variables que dan cuenta de un acceso físico topológico (distancia), un acceso temporal y un acceso funcional (probabilidad funcional) eminentemente social, por propósito e integrada.

Figura $\mathrm{N}^{\mathrm{o}} 1$

Esquema de relación entre el patrón de movilidad y la probabilidad funcional para un propósito $\mathrm{k}$

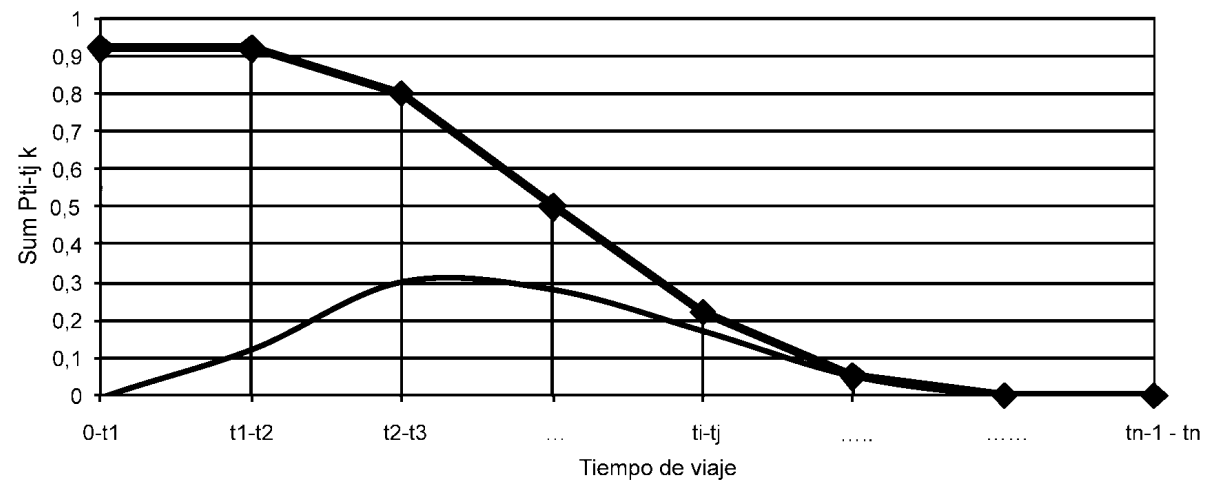

\begin{tabular}{|c|c|c|}
\hline $\begin{array}{l}\text { Intervalo } \\
\text { de tiempo }\end{array}$ & $\begin{array}{l}\text { Patrón de movilidad del } \\
\text { usuario, para el propósito } \mathrm{k}\end{array}$ & $\begin{array}{l}\text { Probabilidad funcional del usuario, } \\
\text { para el propósito } k\end{array}$ \\
\hline $\mathrm{t} 1-\mathrm{t} 2$ & Pt1,t2 k & Ptn -1, tn $k+\ldots \ldots+\ldots+$ Pti,tj $k+\ldots+P t 2, t 3 k+P t 1, t 2 k=1,00$ \\
\hline $\mathrm{t} 2-\mathrm{t} 3$ & Pt2,t3 k & Ptn -1, tn $k+\ldots \ldots+\ldots+$ Pti, tj k $+\ldots+$ Pt2,t3 $k$ \\
\hline$\ldots$ & $\ldots$ & Ptn -1, tn $k+\ldots \ldots+\ldots+$ Pti,tj $k+\ldots$ \\
\hline $\mathrm{ti}-\mathrm{tj}$ & Pti,tj k & Ptn-1,tn $k+\ldots \ldots+\ldots+$ Pti,tj $k$ \\
\hline$\ldots$ & $\ldots$ & Ptn $-1, \operatorname{tn} k+\ldots \ldots+\ldots$ \\
\hline$\ldots$ & $\ldots \ldots$ & Ptn- 1 , tn $k+\ldots \ldots$ \\
\hline tn-1 - tn & Ptn-1, tn k & Ptn-1, tn k \\
\hline Total & 1,00 & \\
\hline
\end{tabular}

Fuente: Elaboración propia. 


\section{Evaluación del poder explicativo de las distintas accesibilidades sobre la estructura espacial de densidad residencial}

Para esto se calcula, con base en la información de los respectivos censos, la densidad poblacional en habitantes por hectárea ${ }^{6}$.

Para analizar el poder explicativo que tienen las medidas de acceso consideradas (tiempo y distancia), y la probabilidad funcional construida, sobre la estructura de densidades de las ciudades, se calibraron modelos econométricos univariados, considerando la densidad poblacional como variable dependiente, y las medidas de acceso (y probabilidad funcional) como variables explicativas (un modelo para cada medida de acceso). Los modelos calibrados, considerando distancia o tiempo, responden a la estructura tradicional de análisis de las densidades, que se basa en la observación empírica de Colin Clark (1951). Clark estructura la función exponencial negativa, originalmente respecto de la distancia, pero más genéricamente, respecto de la accesibilidad. La ecuación de Clark tiene la siguiente estructura:

$$
D_{i}=D * \exp \left(-\beta * A c C_{i}\right)
$$

Donde:

$D_{i} \quad$ : densidad demográfica de la zona $i$.

$D$ : densidad a estimar en el centro considerado.

$\beta \quad$ : parámetro a calibrar, denominado gradiente de densidad.

$A c c_{i}$ : medida de accesibilidad específica de la zona $i$ a la centralidad considerada por propósito e integrada.

El modelo cásico de Clark ha generado una gran cantidad de aplicaciones empíricas en áreas metropolitanas y ciudades (Martori, 2001). Muchas de estas aplicaciones han sido utilizadas en estudios de transporte y de mercado de viviendas. Si bien la formulación de Clark no es la única estructura funcional que puede explicar el comportamiento de la densidad poblacional en el espacio, todas

\footnotetext{
6 Medida de densidad utilizada en Chile.
}

comparten la filosofía exponencial, diferenciando solo la estructura funcional del gradiente (Martori, 2001). Así, el modelo de Clark resulta ser de fundamento empírico, y no teórico, en la descripción de la estructura espacial de la densidad poblacional.

En el modelo clásico (ecuación 1), la medida de separación espacial (que con la función exponencial se transforma en medida de accesibilidad o fricción espacial) hace referencia a la distancia al centro de la ciudad (CBD). En este caso se consideraron las centralidades más cercanas tanto de viajes atraídos como de locales de actividad económica, para los propósitos considerados. Es decir, se evalúa la capacidad estructurante (ordenadora) que tienen las distintas centralidades cercanas sobre la estructura de densidades poblacionales, y no una única centralidad espacial (monocentro). Por tanto el análisis no parte de un modelo monocéntrico, sino policéntrico, a diferencia de las distintas aplicaciones que se han realizado a estructuras policéntricas, en las cuales se ha calibrado el modelo para cada subcentro (considerándolo como monocentro) por separado (Martori, 2001).

Por otra parte, la consideración del tiempo como variable de separación espacial (y no la distancia) es coherente con la filosofía y formulación clásica del modelo, a pesar de existir poca literatura al respecto.

Los modelos calibrados, considerando la probabilidad funcional, tienen otra estructura matemática, dado el concepto y forma de cálculo del indicador (su máximo valor 1 se da en el subcentro). La estructura de estos modelos es la siguiente:

$$
D_{i}=\beta * P f_{i}
$$

Donde:

$D_{i}$ : densidad demográfica de la zona $i, y$ también densidad de inmigración de la zona $i$.

$\beta$ : parámetro a calibrar, denominado gradiente de densidad, que en este caso representa también la densidad en el centro considerado.

$P f_{i}$ : probabilidad funcional por propósito e integrada, de la zona $i$ respecto de las centralidades consideradas. 
Finalmente se calibraron un total de 12 modelos por ciudad (cuatro propósitos y tres medidas de accesibilidad), por año. Los resultados de la calibración se validaron bajo los supuestos econométricos comunes, relacionados con la coherencia de signos, la significancia estadística de los parámetros, la distribución de los residuos, para finalmente evaluar el ajuste del modelo en base al coeficiente de determinación $\left(\mathrm{R}^{2}\right)$ ajustado.

Si bien las estructuras funcionales calibradas son distintas, ambas responden a la filosofía y suposición de la existencia de un gradiente de densidad en torno a cada subcentro detectado (centralidad por propósito). Las diferentes estructuras funcionales hacen que los parámetros obtenidos no sean comparables. Lo que sí se puede comparar es el poder explicativo de cada modelo, pues es necesario recordar que el objetivo de este estudio no es encontrar el mejor modelo, sino verificar en qué medida la densidad poblacional se explica por las distintas medidas tradicionales de acceso en el territorio versus la probabilidad funcional construida.

\section{Resultados}

\section{Comportamiento de los viajes y sus propósitos}

Para el trabajo lo importante es caracterizar los propósitos de los viajes, dado que estos son un buen reflejo de cómo funciona la ciudad, en términos de las interacciones necesarias para satisfacer las necesidades de la población y de las actividades. En el Cuadro $\mathrm{N}^{\circ} 2$ se muestran los valores agregados para ambas ciudades.

En el Cuadro $N^{\circ} 2$ se aprecia que para la ciudad de Santiago el número de viajes aumenta significativamente en el período 1991-2001 (aproximadamente un 80\%), incrementando la cantidad de viajes en todos los propósitos analizados. Este aumento de la

\section{Cuadro $\mathrm{N}^{\circ} 2$}

Estructura de la movilidad por propósitos y su evolución

\begin{tabular}{|c|c|c|c|c|c|}
\hline \multicolumn{6}{|c|}{ Santiago } \\
\hline \multirow{2}{*}{ Propósito del viaje } & \multicolumn{2}{|l|}{1991} & \multicolumn{2}{|l|}{2001} & \multirow{2}{*}{ Diferencia 2001-1991 (\%) } \\
\hline & Viajes por día & $\%$ & Viajes por día & $\%$ & \\
\hline Estudio & 2.720 .457 & 32 & 3.168 .083 & 21 & $-11,42$ \\
\hline Trabajo & 3.481 .094 & 42 & 4.135 .473 & 28 & $-14,08$ \\
\hline Compras & 1.504 .803 & 18 & 4.556 .111 & 30 & 12,35 \\
\hline Otros & 660.177 & 8 & 3.161 .350 & 21 & 13,16 \\
\hline Total & 8.366 .531 & 100 & 15.021 .017 & 100 & 0,00 \\
\hline \multicolumn{6}{|c|}{ Barcelona } \\
\hline \multirow[b]{2}{*}{ Propósito del viaje } & \multicolumn{2}{|l|}{1996} & \multicolumn{2}{|l|}{2001} & \multirow[b]{2}{*}{ Diferencia 2001-1991 (\%) } \\
\hline & $\begin{array}{l}\text { Viajes por } \\
\text { semana }\end{array}$ & $\%$ & $\begin{array}{l}\text { Viajes por } \\
\text { semana }\end{array}$ & $\%$ & \\
\hline Estudio & 3.075 .520 & 14 & 4.881 .914 & 19 & 4,38 \\
\hline Trabajo & 8.688 .416 & 41 & 9.106 .379 & 35 & $-5,55$ \\
\hline Compras & 2.358 .181 & 11 & 3.010 .680 & 11 & 0,56 \\
\hline Otros & 7.384 .165 & 34 & 9.129 .160 & 35 & 0,61 \\
\hline Total & 21.506 .283 & 100 & 26.128 .133 & 100 & 0,00 \\
\hline
\end{tabular}

Fuentes: Encuestas Origen Destino (SECTRA, 1991 y 2001); Encuesta de Movilidad Cotidiana (ATM, 1996 y 2001 ). 
movilidad se debe, en parte, al crecimiento demográfico, y preferentemente al aumento (relativo) de las rentas de clases bajas, incorporando a esta población como nuevos nichos de demanda de bienes de consumo, en el marco del acelerado crecimiento económico del país.

Respecto a la partición por propósitos, se aprecia un fuerte cambio estructural, ya que la movilidad ocupacional (trabajo y estudio, denominada también como movilidad obligada) pierde participación del año 1991 al 2001, en relación al significativo aumento de la movilidad personal (denominada también como movilidad no obligada), tanto para el propósito compras, como para otros como salud, ocio, entretención, visitas sociales. Finalmente en el año 2001 se igualan las particiones de propósitos obligados con no obligados.

En la ciudad de Barcelona, la estructura de propósitos no cambia significativamente entre los años 1996 y 2001. Se aprecia que la movilidad ocupacional pierde un punto porcentual respecto de la personal. Este punto surge del aumento, por una parte, del propósito estudios y de la disminución mayor de la partición del propósito trabajo. La movilidad personal mantiene su concentración preferentemente en el propósito otros (34\%), y en menor porcentaje, en el propósito compras $(12 \%)$.

\section{Patrón de movilidad de los usuarios y probabilidad funcional}

Para caracterizar la distribución estadística de los patrones de movilidad se han utilizado percentiles en vez de promedios, debido a que las distribuciones son claramente asimétricas, por lo que el valor promedio no es una buena medida de tendencia central. El análisis de la distribución de valores permite diferenciar los viajes más cortos, los más largos y los medios. Entonces, se calcularon los percentiles 10, 20, 40, 50, 60, 80 y 90, con el fin de tener una imagen de buena resolución de todas las situaciones, en lo referido al tiempo y distancia de viaje.

En el caso de Barcelona, para cada viaje reportado en la base original de la encuesta EMQ 2001, se obtuvo la distancia de viaje referida a las secciones censales de origen y destino del mismo (recogidas en la encuesta). Para esto se calculó la ruta mínima, a través de la red vial, entre los centroides de las correspondientes secciones censales. En el caso de Santiago, la distancia se determina de la misma forma, pero considerando las zonas de transporte.

Los resultados obtenidos para el año 2001 en ambas ciudades, se presentan en el Cuadro $\mathrm{N}^{\circ} 3$.

En el Cuadro $\mathrm{N}^{\circ} 3$ se pueden ver los valores para los distintos percentiles de distancia de viaje $(\mathrm{km})$ y de tiempo de viaje $(\mathrm{min})$, y además la diferencia entre Barcelona (BCN) y Santiago (STG), para el año 2001. A modo de ejemplo se aprecia que el $80 \%$ de los viajes en Santiago, en el año 2001 (percentil 80 ), tienen una longitud hasta $8,4 \mathrm{~km}$ para el propósito estudio, $15,8 \mathrm{~km}$ para el propósito trabajo y $6,7 \mathrm{~km}$ para compras.

Para ambas ciudades, tanto las distancias como los tiempos del propósito compras son significativamente menores que los valores del propósito estudio y trabajo. El propósito trabajo es el que más se extiende, en ambas ciudades, tanto en distancia como en tiempo de viaje. Lo anterior es coherente con la denominación ocupacional (por ejemplo destino cautivo).

En distancias de viaje, Barcelona presenta (para todos los percentiles) viajes más cortos que Santiago. En general esta diferencia es menor en los viajes más cortos y mayor en los viajes más largos, siendo los de Barcelona aproximadamente la mitad de la longitud de los de Santiago.

Analizando los tiempos de viaje del propósito estudio, se aprecia que en Barcelona se viaja menos tiempo que en Santiago, siendo las diferencias del orden del 12\% (aproximadamente tres minutos). En este caso ya no solo los viajes más cortos son los distintos, la diferencia es relativamente constante en viajes medios y largos. Por el propósito trabajo en Barcelona se viaja significativamente menos que en Santiago, presentando mayores diferencias los viajes largos (del orden del $45 \%$ ). Finalmente, en el propósito compras, es Santiago la ciudad que presenta menores valores, aunque las diferencias son 
porcentualmente menores (del orden del $7 \%$ ). En este caso se puede plantear la igualdad (estadística) de los patrones entre ambas ciudades. En lo específico se aprecia que Santiago tiene menores tiempos en los viajes más cortos, pero en los viajes más largos es Barcelona la que presenta valores menores que Santiago.

La lectura de los resultados muestra que Santiago se caracteriza por una estructura monocéntrica de empleo, y dispersión comercial y educacional. Barcelona, en general, tiene una estructura policéntrica (de ahí los viajes comparativamente más cortos en distancia y tiempo). Lo anterior refleja la similitud estadística en los patrones del propósito compras y estudio, y la significativa diferencia en el patrón de trabajo.

Con los patrones de movilidad identificados, se construyeron las distribuciones empíricas de probabilidad funcional para ambas ciudades (Figura $\mathrm{N}^{\circ} 2$ ).

En la Figura $\mathrm{N}^{\circ} 2$ se presenta la tabla empírica obtenida, con la probabilidad funcional según tiempo de viaje. La lectura de esta tabla indica, por ejemplo, que si un territorio se encuentra a 32 minutos de viaje de una centralidad, existe una probabilidad de 0,15 de que se efectúe el viaje de estudio a dicha centralidad, y de 0,10 si el propósito es compras. Así, el territorio

\section{Cuadro $\mathrm{N}^{\circ} 3$}

Comparación de los patrones de movilidad temporal y espacial

\begin{tabular}{|c|c|c|c|c|c|c|c|c|c|}
\hline \multicolumn{10}{|c|}{ Distancia de viaje } \\
\hline Año 2001 & \multicolumn{3}{|c|}{ Viaje a estudio } & \multicolumn{3}{|c|}{ Viaje a trabajo } & \multicolumn{3}{|c|}{ Viaje a compras } \\
\hline Percentil & $\begin{array}{l}\mathrm{BCN} \\
(\mathrm{km})\end{array}$ & $\begin{array}{l}\text { STG } \\
(\mathrm{km})\end{array}$ & $\begin{array}{c}\text { BCN-STG } \\
(\mathrm{km})\end{array}$ & $\begin{array}{l}\mathrm{BCN} \\
(\mathrm{km})\end{array}$ & $\begin{array}{l}\text { STG } \\
(\mathrm{km})\end{array}$ & $\begin{array}{c}\text { BCN-STG } \\
(\mathrm{km})\end{array}$ & $\begin{array}{l}\mathrm{BCN} \\
(\mathrm{km})\end{array}$ & $\begin{array}{l}\text { STG } \\
(\mathrm{km})\end{array}$ & $\begin{array}{c}\text { BCN-STG } \\
(\mathrm{km})\end{array}$ \\
\hline 10 & 0,4 & 0,5 & $-0,1$ & 1,0 & 1,1 & 0,0 & 0,3 & 0,3 & 0,0 \\
\hline 20 & 0,8 & 0,9 & $-0,1$ & 1,4 & 2,4 & $-1,0$ & 0,6 & 0,6 & 0,1 \\
\hline 40 & 1,4 & 2,1 & $-0,6$ & 2,5 & 6,0 & $-3,5$ & 1,3 & 1,2 & 0,0 \\
\hline 50 & 1,7 & 2,9 & $-1,2$ & 3,5 & 8,1 & $-4,6$ & 1,6 & 1,8 & $-0,2$ \\
\hline 60 & 2,0 & 4,2 & $-2,2$ & 4,7 & 10,4 & $-5,7$ & 1,9 & 2,7 & $-0,8$ \\
\hline 80 & 5,0 & 8,4 & $-3,4$ & 8,7 & 15,8 & $-7,1$ & 3,7 & 6,7 & $-3,0$ \\
\hline 90 & 9,5 & 12,3 & $-2,9$ & 13,8 & 20,5 & $-6,6$ & 6,3 & 10,3 & $-4,0$ \\
\hline \multicolumn{10}{|c|}{ Tiempo de viaje } \\
\hline Año 2001 & \multicolumn{3}{|c|}{ Viaje a estudio } & \multicolumn{3}{|c|}{ Viaje a trabajo } & \multicolumn{3}{|c|}{ Viaje a compras } \\
\hline Percentil & $\begin{array}{l}\mathrm{BCN} \\
(\mathrm{min})\end{array}$ & $\begin{array}{l}\text { STG } \\
(\min )\end{array}$ & $\begin{array}{c}\text { BCN-STG } \\
(\min )\end{array}$ & $\begin{array}{l}\mathrm{BCN} \\
(\mathrm{min})\end{array}$ & $\begin{array}{l}\text { STG } \\
(\min )\end{array}$ & $\begin{array}{c}\text { BCN-STG } \\
(\mathrm{min})\end{array}$ & $\begin{array}{l}\mathrm{BCN} \\
(\mathrm{min})\end{array}$ & $\begin{array}{l}\text { STG } \\
(\min )\end{array}$ & $\begin{array}{c}\text { BCN-STG } \\
(\mathrm{min})\end{array}$ \\
\hline 10 & 6,0 & 5,3 & 0,7 & 6,9 & 6,8 & 0,1 & 5,8 & 2,6 & 3,2 \\
\hline 20 & 8,2 & 8,0 & 0,2 & 10,1 & 12,0 & $-1,9$ & 7,5 & 5,1 & 2,4 \\
\hline 40 & 12,2 & 13,6 & $-1,4$ & 15,4 & 23,2 & $-7,8$ & 11,1 & 9,1 & 2,0 \\
\hline 50 & 14,0 & 16,9 & $-2,9$ & 19,7 & 28,6 & $-8,9$ & 13,3 & 11,5 & 1,8 \\
\hline 60 & 17,0 & 21,0 & $-3,9$ & 26,2 & 36,6 & $-10,5$ & 15,8 & 14,3 & 1,5 \\
\hline 80 & 28,4 & 33,1 & $-4,7$ & 30,3 & 56,7 & $-26,3$ & 27,1 & 25,8 & 1,3 \\
\hline 90 & 42,6 & 45,7 & $-3,0$ & 44,9 & 73,2 & $-28,3$ & 32,4 & 37,3 & $-4,9$ \\
\hline
\end{tabular}

BCN: Barcelona; STG: Santiago.

Fuente: Elaboración propia. 
Figura $N^{\circ} 2$

Probabilidad funcional por propósito para Barcelona (2001)

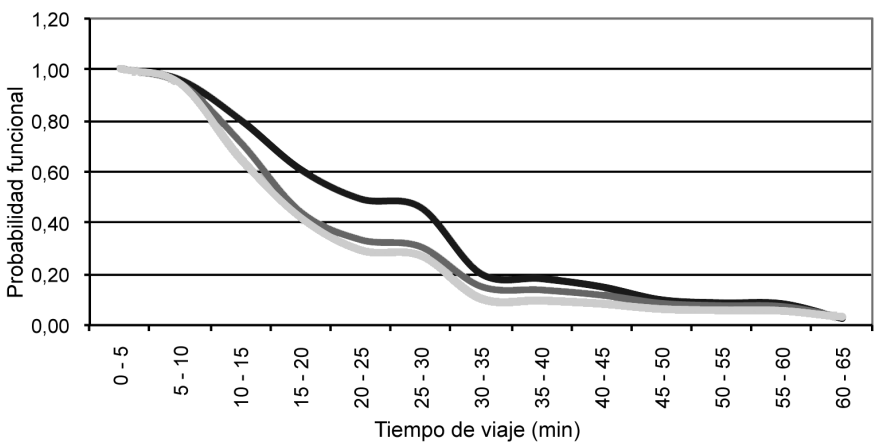

- Viaje a trabajo $\longrightarrow$ Viaje a estudio $\longrightarrow$ Viaje a compras

\begin{tabular}{|c|c|c|c|}
\hline \multirow[b]{2}{*}{ Tiempo de viaje (min) } & \multicolumn{3}{|c|}{ Probabilidad funcional } \\
\hline & Viaje a trabajo & Viaje a estudio & Viaje a compras \\
\hline $0-5$ & 1,00 & 1,00 & 1,00 \\
\hline $5-10$ & 0,96 & 0,95 & 0,95 \\
\hline $10-15$ & 0,80 & 0,72 & 0,65 \\
\hline $15-20$ & 0,61 & 0,44 & 0,42 \\
\hline $20-25$ & 0,49 & 0,33 & 0,29 \\
\hline $25-30$ & 0,46 & 0,31 & 0,27 \\
\hline $30-35$ & 0,20 & 0,15 & 0,10 \\
\hline $35-40$ & 0,18 & 0,14 & 0,10 \\
\hline $40-45$ & 0,15 & 0,12 & 0,08 \\
\hline $45-50$ & 0,10 & 0,08 & 0,06 \\
\hline $50-55$ & 0,09 & 0,08 & 0,06 \\
\hline $55-60$ & 0,08 & 0,07 & 0,06 \\
\hline $60-65$ & 0,03 & 0,03 & 0,03 \\
\hline $65-70$ & 0,02 & 0,03 & 0,03 \\
\hline $70-75$ & 0,02 & 0,03 & 0,03 \\
\hline $75-80$ & 0,02 & 0,02 & 0,03 \\
\hline $80-85$ & 0,02 & 0,02 & 0,02 \\
\hline $85-90$ & 0,01 & 0,02 & 0,02 \\
\hline $90-95$ & 0,01 & 0,01 & 0,02 \\
\hline $95-100$ & 0,01 & 0,01 & 0,02 \\
\hline $100-105$ & 0,01 & 0,01 & 0,02 \\
\hline $105-110$ & 0,01 & 0,01 & 0,02 \\
\hline $110-115$ & 0,01 & 0,01 & 0,01 \\
\hline $115-120$ & 0,01 & 0,01 & 0,01 \\
\hline 120 y más & 0,00 & 0,01 & 0,01 \\
\hline
\end{tabular}

Fuente: Elaboración propia. 
toma atributos en función del tiempo que lo separa del punto en el que se ofrece una actividad (servicio o trabajo) específica, pero condicionado por la disposición a viajar de las personas.

En el gráfico de la Figura $N^{\circ} 2$ se puede ver que los viajes de compras y estudios tienen la mayor inelasticidad en relación a los viajes al trabajo, es decir, hay una menor disposición al tiempo de viaje. El propósito trabajo es más elástico al tiempo de viaje, es decir, las personas están dispuestas a viajar más tiempo. Las mayores diferencias entre propósitos se originan en los tiempos medios (mayor desigualdad entre las curvas). El punto de inflexión más claro, para todos los propósitos, se presenta en los 30 a 35 minutos de viaje. Hasta dicho tiempo de viaje, las curvas decaen rápidamente, para luego mantener una probabilidad baja relativamente constante.

Para Santiago (Figura $N^{\circ} 3$ ) se aprecian mayores diferencias entre propósitos. Aparece el propósito trabajo mucho más elástico al tiempo, resultando la gráfica prácticamente como una línea recta (sin punto de inflexión claro). El propósito estudio es más inelástico al tiempo, mostrando un punto de inflexión entre 40 a 45 minutos. Finalmente las compras presenta mayor inelasticidad, y un punto de inflexión similar que Barcelona, entre los 30 y 35 minutos.

Al comparar las dos ciudades, los comportamientos entre propósitos son similares, pero con valores menores en Barcelona. Siguiendo con el ejemplo del viaje de 32 minutos planteado para Barcelona, en Santiago se tiene una mayor probabilidad de realización si es de trabajo $(0,47$ versus 0,20 para Barcelona), también es mayor si es de estudio $(0,23$ versus 0,15$)$, y un valor más similar a Barcelona si es de compras $(0,15$ versus 0,10$)$. Luego hay patrones muy distintos, como el propósito trabajo, y relativamente similares como los propósitos educación y compras.

\section{Centralidades territoriales}

Como se ha explicado en la metodología, las centralidades se determinaron como las zonas cuyas densidades están en el 25\% de valores más altos en términos de viajes/ha, y en locales/ha, asociados al propósito $k$. En las Figuras $\mathrm{N}^{\circ} 4$ y $\mathrm{N}^{\circ} 5$ se muestra el resultado de esta identificación solo para el propósito compras en ambas ciudades.

En Santiago se aprecia una mayor dispersión de las centralidades tanto en locales como en viajes, mientras que en Barcelona se aprecia una concentración de locales y una dispersión relativa en los viajes atraídos.

Además, se observa un relativo desfase espacial entre las mayores concentraciones de locales y las mayores atracciones de viajes. $\mathrm{Si}$ bien este trabajo no tiene por objeto analizar estas diferencias, se genera una interrogante de investigación, en relación a que siempre se ha considerado como supuesto base que donde existe mayor oferta de actividades se atraerá la mayor cantidad de viajes.

\section{Evaluación de la capacidad explicativa de las distintas accesibilidades y la estructura espacial de densidad residencial}

A continuación (Figura $N^{\circ}$ 6) se muestra la conformación de densidades poblacionales de Barcelona y Santiago para el año 2001 (escalas de densidades comparables), con el fin de tener una imagen de la estructura urbana que se quiere explicar.

Se aprecia en Santiago una estructura periférica de las altas densidades, que a su vez son significativamente menores en magnitud que Barcelona, donde se percibe una estructura concentrada y central, que se extiende en dirección a algunos ejes estructurantes.

En los Cuadros $\mathrm{N}^{\circ} 4$ y $\mathrm{N}^{\circ} 5$ se presentan los resultados de las calibraciones de los modelos econométricos que buscan explicar la estructura de las densidades. Se exponen, por modelo, los valores obtenidos de la constante (cuando corresponda), del coeficiente beta (gradiente de densidad en distancia y tiempo, y densidad central para probabilidad funcional) y el grado de ajuste logrado (proporción de explicación de los valores observados por parte del modelo). 
Figura $\mathrm{N}^{\circ} 3$

Probabilidad funcional por propósito para Santiago (2001)

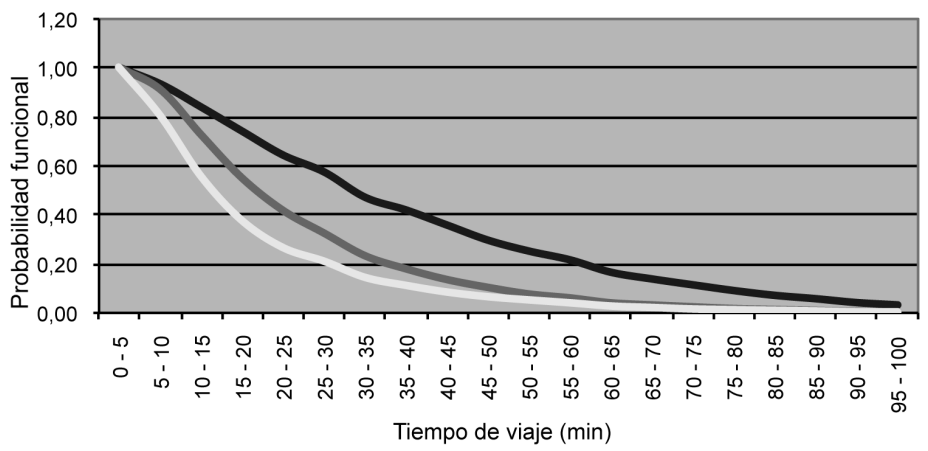

\begin{tabular}{|l}
- Viaje a trabajo $\longrightarrow$ Viaje a estudio $\longrightarrow$ Viaje a compras \\
\hline
\end{tabular}

\begin{tabular}{|c|c|c|c|}
\hline \multirow[b]{2}{*}{ Tiempo de viaje (min) } & \multicolumn{3}{|c|}{ Probabilidad funcional } \\
\hline & Viaje a trabajo & Viaje a estudio & Viaje a compras \\
\hline $0-5$ & 1,00 & 1,00 & 1,00 \\
\hline $5-10$ & 0,93 & 0,91 & 0,80 \\
\hline $10-15$ & 0,84 & 0,73 & 0,56 \\
\hline $15-20$ & 0,74 & 0,55 & 0,38 \\
\hline $20-25$ & 0,64 & 0,42 & 0,27 \\
\hline $25-30$ & 0,58 & 0,33 & 0,21 \\
\hline $30-35$ & 0,47 & 0,23 & 0,15 \\
\hline $35-40$ & 0,42 & 0,18 & 0,11 \\
\hline $40-45$ & 0,36 & 0,14 & 0,09 \\
\hline $45-50$ & 0,30 & 0,10 & 0,07 \\
\hline $50-55$ & 0,25 & 0,08 & 0,05 \\
\hline $55-60$ & 0,22 & 0,06 & 0,04 \\
\hline $60-65$ & 0,17 & 0,04 & 0,03 \\
\hline $65-70$ & 0,14 & 0,03 & 0,02 \\
\hline $70-75$ & 0,11 & 0,02 & 0,02 \\
\hline $75-80$ & 0,09 & 0,02 & 0,01 \\
\hline $80-85$ & 0,07 & 0,01 & 0,01 \\
\hline $85-90$ & 0,06 & 0,01 & 0,01 \\
\hline $90-95$ & 0,04 & 0,01 & 0,01 \\
\hline $95-100$ & 0,03 & 0,01 & 0,00 \\
\hline $100-105$ & 0,03 & 0,00 & 0,00 \\
\hline $105-110$ & 0,02 & 0,00 & 0,00 \\
\hline $110-115$ & 0,01 & 0,00 & 0,00 \\
\hline $115-120$ & 0,01 & 0,00 & 0,00 \\
\hline 120 y más & 0,01 & 0,00 & 0,00 \\
\hline
\end{tabular}

Fuente: Elaboración propia. 


\section{Figura $\mathrm{N}^{\circ} 4$}

Centralidades (25\% de mayores valores) para la ciudad de Santiago (2001)

(a) Densidad de viajes de compras

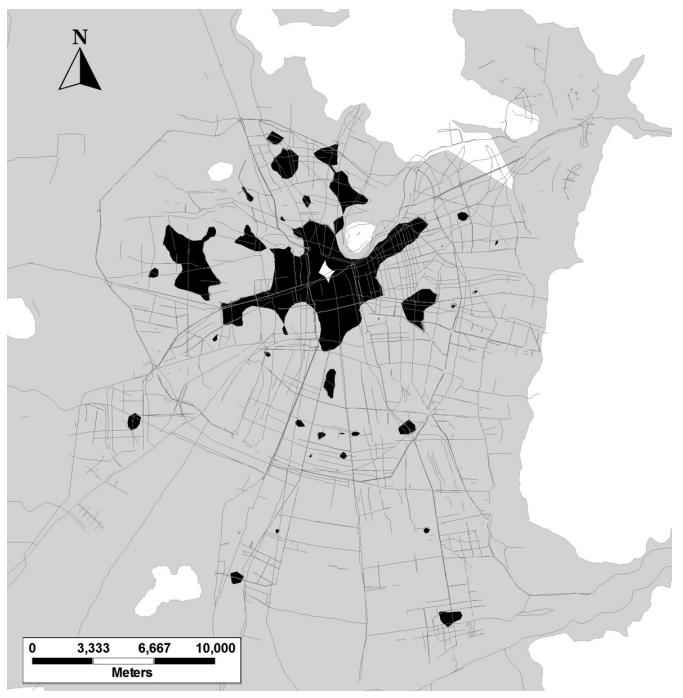

(b) Densidad de locales comerciales

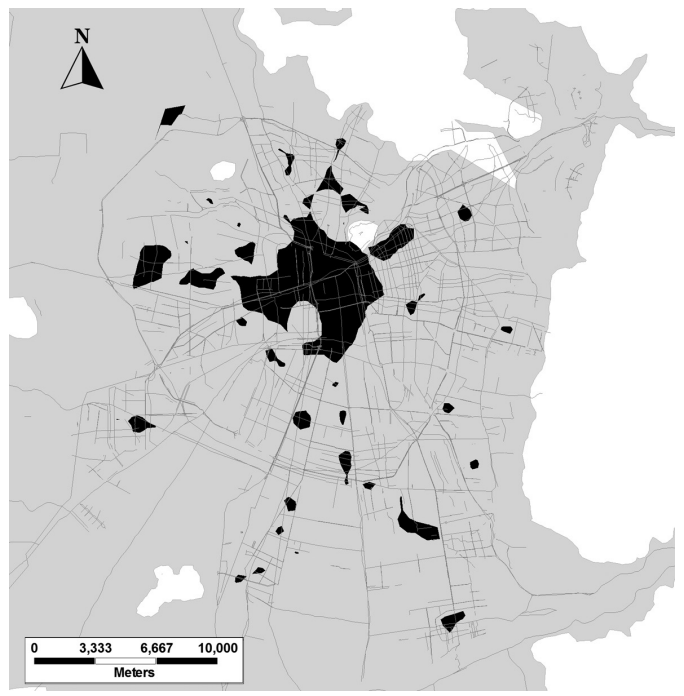

Fuente: Elaboración propia.

Figura $\mathrm{N}^{0} 5$

Centralidades (25\% de mayores valores) para la ciudad de Barcelona (2001) (a) Densidad de viajes de compras

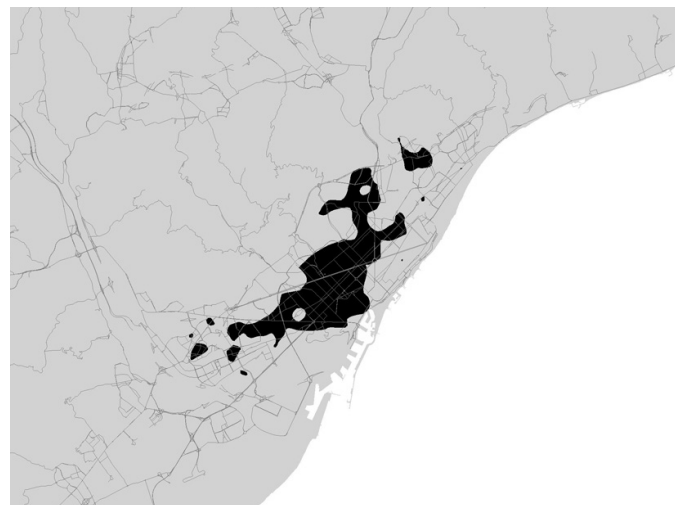

(b) Densidad de locales comerciales

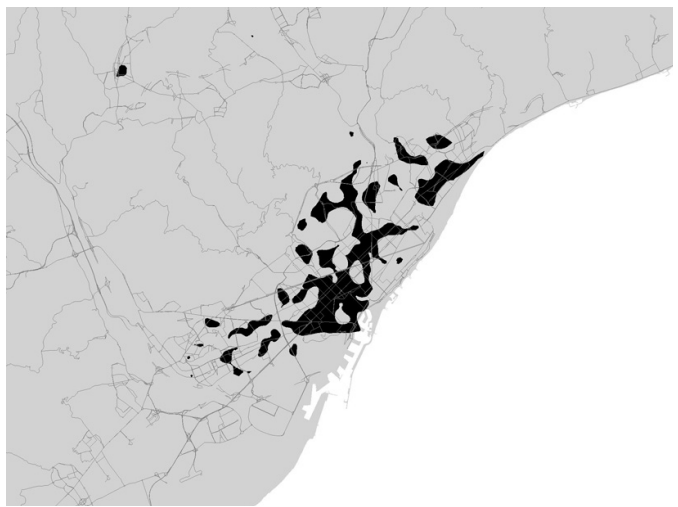

Fuente: Elaboración propia. 
Figura $N^{\circ} 6$

Densidades de población 2001

(a) Santiago

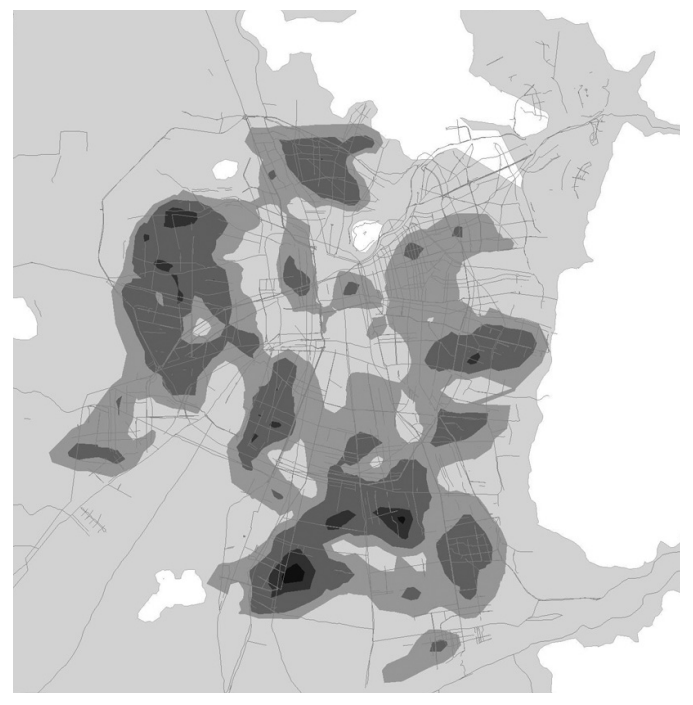

Fuente: Elaboración propia.

Los modelos para Santiago (Cuadro $\mathrm{N}^{\circ}$ 4), en el año 1991, muestran que la distancia y tiempos de viaje a las centralidades más cercanas no explican de manera importante (ajustes bajo el 0,2) la estructura de densidades poblacionales, bajo el supuesto de gradiente exponencial. Lo anterior contrasta con el alto grado de explicación (sobre el $0,75)$ que tiene la probabilidad funcional, tanto por propósitos, como para la probabilidad funcional integrada (composición de las probabilidades por propósito ponderadas por su partición en el total de viajes). Ello sugiere que la probabilidad funcional, con independencia que se refiera a las centralidades de locales o a los destinos de los viajes, explica (estadísticamente hablando) la estructura espacial de la densidad residencial de la ciudad de Santiago, en el año 1991.

Santiago, en el año 2001 (Cuadro $N^{\circ}$ 5), muestra una mejora significativa de las medidas de accesibilidad tradicional, en lo que se refiere a explicación de las densidades poblacionales (valores superiores a 0,35). A pesar de esto, sigue siendo la probabilidad funcional la variable de mejor poder explicativo de la estructura espacial de las densidades, con (b) Barcelona

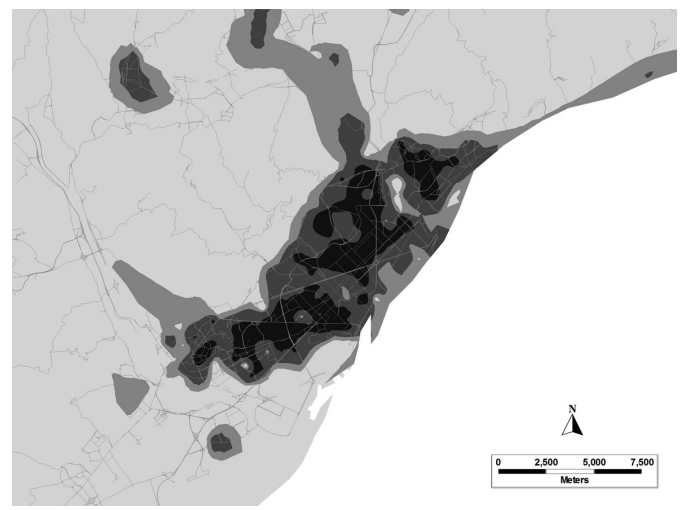

un ajuste muy superior a los obtenidos por distancia y/o tiempo (mayores que 0,7 ).

Los gradientes de densidad calibrados (modelos de distancia y tiempo), resultan ser relativamente constantes, independiente del propósito que se analice y del tipo de centralidad considerado.

Al comparar los valores obtenidos con estudios desarrollados por Echeñique (2006) para Santiago a nivel de municipios (donde se obtiene un $D=116 ; \beta=-0,03 ; R^{2}=0,51$ considerando la distancia al centro), se aprecia que el gradiente de densidad respecto de múltiples centralidades es mucho mayor que el obtenido respecto del CBD, lo que indica un superior poder de estructuración de las centralidades funcionales, que del centro histórico de la ciudad.

Los modelos para Barcelona, en el año 2001 (Cuadro $N^{\circ}$ 6), muestran una mayor capacidad de explicación de la distancia y tiempo, que en el caso de Santiago. Así, los modelos de distancia logran una explicación del orden del 57\% (valores superiores a 0,54$)$, mientras que los modelos de tiempo 
Cuadro $\mathrm{N}^{\circ} 4$

Resultados calibración modelos econométricos para Santiago (1991)

\begin{tabular}{|c|c|c|c|c|c|c|c|c|}
\hline \multirow{3}{*}{ Subcentro considerado } & \multicolumn{8}{|c|}{ Tipo de variable de accesibilidad (x) utilizada en el modelo } \\
\hline & \multicolumn{3}{|c|}{ Distancia $(\mathrm{km})$} & \multicolumn{3}{|c|}{ Tiempo (min) } & \multicolumn{2}{|c|}{$\begin{array}{l}\text { Probabilidad } \\
\text { funcional }\end{array}$} \\
\hline & Cte & Beta & Adj. R2 & Cte & Beta & Adj. R2 & Beta & Adj. R2 \\
\hline Densidad locales de trabajo & 4,580 & $-0,104$ & 0,07 & 4,742 & $-0,023$ & 0,03 & 110,6 & 0,80 \\
\hline Densidad locales de estudio & 4,560 & $-0,112$ & 0,05 & ns & ns & - & 122,4 & 0,73 \\
\hline Densidad locales de compras & 4,674 & $-0,190$ & 0,16 & 4,786 & $-0,028$ & 0,03 & 127,0 & 0,77 \\
\hline Acc. integrada subc. locales & 4,656 & $-0,162$ & 0,10 & 4,749 & $-0,024$ & 0,02 & 119,6 & 0,79 \\
\hline Densidad viajes de trabajo & 4,648 & $-0,163$ & 0,13 & 4,567 & $-0,012$ & 0,01 & 108,1 & 0,79 \\
\hline Densidad viajes de estudio & 4,693 & $-0,200$ & 0,25 & 4,875 & $-0,034$ & 0,04 & 118,8 & 0,78 \\
\hline Densidad viajes de compras & 4,674 & $-0,194$ & 0,17 & 4,715 & $-0,022$ & 0,03 & 124,7 & 0,76 \\
\hline Acc. integrada subc. viajes & 4,713 & $-0,211$ & 0,21 & 4,772 & $-0,026$ & 0,02 & 116,2 & 0,79 \\
\hline Variable dependiente (Y) & \multicolumn{3}{|c|}{$\begin{array}{l}\text { Ln (Densidad } \\
\text { poblacional*) }\end{array}$} & \multicolumn{3}{|c|}{$\begin{array}{l}\text { Ln (Densidad } \\
\text { poblacional*) }\end{array}$} & \multicolumn{2}{|c|}{$\begin{array}{c}\text { Densidad } \\
\text { poblacional* }\end{array}$} \\
\hline
\end{tabular}

*Ha/hab.

Fuente: Elaboración propia.

Cuadro $\mathrm{N}^{\circ} 5$

Resultados calibración modelos econométricos Santiago (2001)

\begin{tabular}{|l|c|c|c|c|c|c|c|c|}
\cline { 2 - 8 } \multicolumn{1}{c|}{} & \multicolumn{5}{c|}{ Tipo de variable de accesibilidad (x) utilizada en el modelo } \\
\hline \multirow{2}{*}{ Subcentro considerado } & \multicolumn{3}{|c|}{ Distancia $(\mathrm{km})$} & \multicolumn{3}{c|}{ Tiempo (min) } & \multicolumn{2}{c|}{$\begin{array}{c}\text { Probabilidad } \\
\text { funcional }\end{array}$} \\
\cline { 2 - 9 } & Cte & Beta & Adj. R2 & Cte & Beta & Adj. R2 & Beta & Adj. R2 \\
\hline Densidad locales de trabajo & 4,530 & $-0,158$ & 0,41 & 4,479 & $-0,044$ & 0,30 & 91,7 & 0,77 \\
Densidad locales de estudio & 4,482 & $-0,164$ & 0,38 & 4,453 & $-0,044$ & 0,27 & 101,5 & 0,70 \\
Densidad locales de compras & 4,486 & $-0,171$ & 0,42 & 4,461 & $-0,047$ & 0,31 & 102,4 & 0,75 \\
Acc. integrada subc. locales & 4,553 & $-0,180$ & 0,45 & 4,527 & $-0,051$ & 0,33 & 101,3 & 0,77 \\
Densidad viajes de trabajo & 4,494 & $-0,168$ & 0,38 & 4,487 & $-0,048$ & 0,28 & 90,8 & 0,76 \\
Densidad viajes de estudio & 4,480 & $-0,184$ & 0,48 & 4,476 & $-0,052$ & 0,34 & 94,6 & 0,78 \\
Densidad viajes de compras & 4,469 & $-0,178$ & 0,44 & 4,420 & $-0,047$ & 0,29 & 99,7 & 0,74 \\
Acc. integrada subc. viajes & 4,517 & $-0,188$ & 0,46 & 4,519 & $-0,054$ & 0,33 & 97,1 & 0,78 \\
\hline Variable dependiente (Y) & \multicolumn{3}{|c|}{ Ln (Densidad } & Ln (Densidad & Densidad \\
\hline
\end{tabular}

*Ha/hab.

Fuente: Elaboración propia. 
explican del orden de $60 \%$ de la variabilidad de la densidad poblacional. En este caso, la probabilidad funcional también tiene un alto grado de ajuste en la explicación de la densidad (valores superiores a 0,6), siendo levemente mayor que los ajustes con distancia y con tiempo.

Los resultados de las modelaciones para Santiago y Barcelona denotan tres puntos importantes:

a) La probabilidad funcional es la medida de acceso que mejor explica la distribución espacial de densidades poblacionales, logrando ajustes muy altos (del orden del 0,65 a 0,7 en ambas ciudades), y que en el caso de Santiago, este valor se mantiene alto entre los años 1991 y 2001. Además, la probabilidad funcional tiene mayor poder explicativo en Santiago que en Barcelona $(0,75$ contra 0,65 , en promedio). Las medidas tradicionales de acceso (distancia y tiempo) son comparables (en capacidad explicativa) a la probabilidad funcional solo en la ciudad de Barcelona, aunque Santiago muestra un aumento de la capacidad explicativa tanto de la distancia como del tiempo en el período 1991-2001.

b) Los gradientes de densidad (coeficiente beta) en Barcelona son significativamente mayores que en Santiago, tanto para distancias como para tiempos de viaje, lo que habla de una mayor inelasticidad o tensión en la concentración de densidades en torno a las centralidades detectadas.

c) No se aprecia una clara diferencia entre los modelos con centralidades por locales versus los de centralidades de viajes. Lo anterior mantiene abierta la interrogante de cuáles son las centralidades que ordenan o estructuran de mejor forma las densidades poblacionales, cuestionamiento que no es parte esencial de esta investigación.

Cuadro $\mathrm{N}^{\circ} 6$

Resultados calibración modelos econométricos para Barcelona (2001)

\begin{tabular}{|c|c|c|c|c|c|c|c|c|}
\hline \multirow{3}{*}{ Subcentro considerado } & \multicolumn{8}{|c|}{ Tipo de variable de accesibilidad (x) utilizada en el modelo } \\
\hline & \multicolumn{3}{|c|}{ Distancia $(\mathrm{km})$} & \multicolumn{3}{|c|}{ Tiempo (min) } & \multicolumn{2}{|c|}{$\begin{array}{l}\text { Probabilidad } \\
\text { funcional }\end{array}$} \\
\hline & Cte & Beta & Adj. R2 & Cte & Beta & Adj. R2 & Beta & Adj. R2 \\
\hline Densidad locales de trabajo & 5,147 & $-0,224$ & 0,61 & 5,799 & $-0,192$ & 0,64 & 243,4 & 0,62 \\
\hline Densidad locales de estudio & 5,141 & $-0,189$ & 0,54 & 5,674 & $-0,168$ & 0,56 & 267,0 & 0,67 \\
\hline Densidad locales de compras & 5,141 & $-0,189$ & 0,54 & 5,674 & $-0,168$ & 0,56 & 274,1 & 0,68 \\
\hline Acc. integrada subc. locales & 5,197 & $-0,214$ & 0,60 & 5,797 & $-0,184$ & 0,62 & 254,6 & 0,65 \\
\hline Densidad viajes de trabajo & 5,008 & $-0,225$ & 0,58 & 5,739 & $-0,206$ & 0,66 & 232,9 & 0,60 \\
\hline Densidad viajes de estudio & 4,982 & $-0,225$ & 0,58 & 5,709 & $-0,212$ & 0,66 & 243,7 & 0,63 \\
\hline densidad viajes de compras & 5,058 & $-0,222$ & 0,59 & 5,703 & $-0,198$ & 0,64 & 257,5 & 0,65 \\
\hline Acc. integrada subc. viajes & 5,018 & $-0,224$ & 0,59 & 5,792 & $-0,212$ & 0,68 & 238,9 & 0,62 \\
\hline Variable dependiente (Y) & \multicolumn{3}{|c|}{$\begin{array}{l}\text { Ln (Densidad } \\
\text { poblacional*) }\end{array}$} & \multicolumn{3}{|c|}{$\begin{array}{l}\text { Ln (Densidad } \\
\text { poblacional*) }\end{array}$} & \multicolumn{2}{|c|}{$\begin{array}{c}\text { Densidad } \\
\text { poblacional* }\end{array}$} \\
\hline
\end{tabular}

${ }^{*} \mathrm{Ha}$ /hab.

Fuente: Elaboración propia. 


\section{Consideraciones finales}

El trabajo expuesto logró el objetivo de construir un indicador de acceso funcional y evaluar el grado de explicación que tiene este factor (en relación a los tradicionales de distancia y tiempo) en la densidad poblacional.

Con base en los resultados obtenidos, para dos ciudades demográfica y estructuralmente distintas, se puede concluir que:

- El tiempo y distancia de viaje de los usuarios tiene una distribución estadística, que además no es simétrica, por lo que no es correcta la utilización de la media como medida de caracterización. Cualquier análisis al respecto debe considerar siempre la distribución de los valores, a través de estadígrafos descriptivos.

- Existen patrones de movilidad relativamente similares entre las ciudades (preferentemente en estudio y compras) y claramente distintos para trabajo. Lo primero da cuenta de un incipiente grado de generalidad o transferibilidad de ciertos comportamientos de movilidad urbana.

- En ambas ciudades los resultados sugieren que la movilidad personal tiene una importancia progresivamente creciente. Luego, el hecho que no sea un propósito que estrese las capacidades de infraestructura, no es motivo para que no se estudie, ya no desde un enfoque de ingeniería de transporte, sino más bien desde el punto de vista de gestión urbana (la gestión de los tiempos en la ciudad).

- El concepto de funcionalidad urbana enriquece al tradicional concepto de accesibilidad, al incorporar el comportamiento real de los usuarios, se trata por tanto de una accesibilidad social. Las elasticidades de la disponibilidad a viajar dependen del propósito del mismo, y están claramente diferenciadas. Así se puede plantear una diferenciación entre una disposición a viajar inelástica, asociada a propósitos no obligados versus una elasticidad asociada a propósitos obligados.

- Se puede volver a interpretar el modelo monocéntrico, como el de Clark, consi- derando multicentralidades, y explicar así la estructura de actividades en la ciudad. La condición de monocentrismo no es un requisito de la aplicación de estos modelos, por lo que en su aplicación se pueden incorporar variables de multicentralidad. Esto abre una línea interesante de reinterpretación de modelos monocéntricos, y su validez para ser aplicados en las actuales formas urbanas.

- La estructura de densidades de ambas ciudades se explican, de manera significativa, por alguna de las medidas de acceso considerada. Al respecto, la probabilidad funcional de los territorios es la que resulta ser más explicativa. En base a lo anterior, se demuestra la hipótesis de investigación planteada.

Si bien es aventurado, pero a la luz de los resultados obtenidos en términos de las situaciones de cada ciudad, y los ajustes logrados, se puede plantear otra hipótesis de investigación respecto que en ciudades relativamente compactas, la estructuración espacial de las densidades es determinada indistintamente por tiempos, distancias y/o probabilidades funcionales, debido a que tanto tiempos como distancias están en los rangos probables de la disposición a viajar de los habitantes. En cambio, en ciudades extensas, es solo la probabilidad funcional del territorio la que permite su explicación, dejando de ser explicativos tanto la distancia como el tiempo. Lo anterior debido a que los umbrales de disposición a viajar de distancias y tiempos se han superado, por lo que se conforma una geometría funcional y no física/temporal, es decir, la disposición de viaje de los usuarios condiciona la interacción y la localización.

Sin embargo, para verificar la nueva hipótesis planteada, se requiere aplicar la metodología en más ciudades. Es por eso que este trabajo reporta los primeros resultados de una investigación más amplia, que persigue conocer de mejor forma los patrones de movilidad en la ciudad (entendiendo la ciudad como una composición de sistemas territoriales en donde importan los propósitos de viajes, más que los modos y las infraestructuras), y también investigar en la línea de caracterizar los patrones de tiempo de estadía de los viajeros en las distintas zonas de la ciudad. El 
enfoque es que la movilidad es parte de una secuencia de etapas en la satisfacción de una necesidad, en donde no se puede entender ni estudiar en forma separada del tiempo de estadía en la actividad, y de la información previa de que dispone el individuo.

Con las conclusiones de este trabajo se reafirma la idea de que la capacidad de acceso a determinadas actividades de la ciudad no solo está determinada por las redes de transporte, sino, sobre todo, por el patrón de movilidad de las personas a lo largo de la ciudad, es decir, es un fenómeno de naturaleza social. Los tradicionales paradigmas de causalidad y de capacidad de transporte deben reenfocarse hacia concepciones dialécticas del rol social del transporte en la ciudad, y en definitiva de cómo se condiciona (en parte) el cómo-cuándo-dónde la población utiliza (goza, vive, o es víctima) la ciudad.

\section{Referencias bibliográficas}

AGUILAR, A. Las mega-ciudades y las periferias expandidas. EURE, 2002, vol. 28, $N^{\circ}$ 85, p. 121-149. Disponible en Internet: http://redalyc.uaemex.mx/redalyc/src/inicio/ ArtPdfRed.jsp?iCve=19608507\&iCveNum=0

BATTY, M. Agents, cells and cities: new representational models for simulating multiscale urban dynamics. London: Centre for Advanced Spatial Analysis, Working Paper 65, 2003. Disponible en Internet: http://www. casa.ucl.ac.uk/working_papers.htm

BERRY, B. Urbanization and counterurbanization in the United States. Annals of the American Academy of Political and Social Science, 1980, vol. 451, p. 13-20.

CARUSO, G.; CAVAILHÈS, J.; FRANKHAUSER, P.; PEETERS, D. \& THOMAS, I. TS-GHOST City: self-generating housing, open-space and transportation in the city. In: Boston, Annual Conference of the Association of American Geographers, abril 2008.

CLARK, C. Urban population densities. Journal of the Royal Statistical Society, 1951, vol. 114, p. 490-496. Disponible en Internet: http://www.jstor.org/stable/2981088?seq=4
CERDA, J. El comportamiento del mercado inmobiliario en la cuenca operacional del Gran Santiago: un nuevo enfoque de análisis. En: PEREIRA, P. y HIDALGO, R. (eds.). Producción inmobiliaria y reestructuración metropolitana en América Latina. Santiago: Instituto de Geografía, Pontificia Universidad Católica de Chile, Serie GEOLibros 11, 2008, p. 89-106.

ECHEÑIQUE, M. El crecimiento y el desarrollo de las ciudades. En: GALETOVIC, A. (ed.). Santiago dónde estamos y hacia dónde vamos. Santiago: Centro de Estudios Públicos, 2006, p. 73-96.

ENRÍQUEZ, J. Ciudades de muros. Los fraccionamientos cerrados en la frontera noroeste de México. Sripta Nova, 2007, vol. 11, No 230. Disponible en Internet: http:// www.ub.es/geocrit/sn/sn-230.htm

FLICHY, P. Une histoire de la communication moderne. París: La decouverte, 2004.

GALETOVIC, A. Santiago dónde estamos y hacia dónde vamos. Santiago, Chile: Centro de Estudios Públicos, 2006.

GALSTER, J. et al. Wrestling sprawl to the ground: defining and measuring an elusive concept. Housing Policy Debate, 2001, vol. 12, № 4, p. 681-717. Disponible en Internet: http://www2.gulfofmaine.org/ESIPPlanning/ subcommittees/coastaldevelopment/CoasDevRefDocs/Wrestling $\% 20$ Sprawl $\% 20$ to $\% 20$ the $\% 20$ Ground $\% 20$ Defining $\% 20$ and $\% 20$ Measuring\%20an\%20Elusive\%20Concept.pdf

GARCÍA, J. Incidencia en la movilidad de los principales factores de un modelo metropolitano cambiante. EURE, 2008, vol. 34, $\mathrm{N}^{\circ}$ 101, p. 5-24. Disponible en Internet: http:// www.scielo.cl/pdf/eure/v34n101/art01.pdf

HALL, P. \& HAY, D. Growth centres in the european urban system. London: Heinemann, 1980.

HIDALGO, R.; BORSDORF, A.; ZUNINO, H. y ÁLVAREZ, L. Tipologías de expansión metropolitana en Santiago de Chile: precariópolis estatal y privatópolis inmobiliaria. Scripta Nova. Revista Electrónica de Geografía 
y Ciencias Sociales, 2008, vol. XII, No 270. Disponible en Internet: http://www.ub.es/geocrit/sn/sn-270/sn-270-113.htm

HOYOS, G. La periferia mexiquense en la apertura de la economía urbana de la ciudad de México. Papeles de población, 2000, № 23, p. 71-92. Disponible en Internet: http:// redalyc.uaemex.mx/redalyc/src/inicio/ArtPdfRed.jsp?iCve $=11202304 \& i C v e N u m=0$

KAUFMANN, V. Motility: mobility as capital. International Journal of Urban and Regional Research, 2004, vol. 28, No 4, p. 745-756. Disponible en Internet: http:// www3.interscience.wiley.com/cgi-bin/fulltext/118779126/PDFSTART

LAUTSO, K. Integrated modeling in Europe with PROPOLIS. In: Portland, Fourth Oregon Symposium on Integrated Land-Use Transport Models, November 2005.

MARTÍNEZ, F. Access, the economic link in land use transport interaction. In: Valle Nevado, International Conference on Travel Behaviour Research, octubre 1994.

MARTORI, J. \& SURIÑACH, J. Classical models of urban population density. The case of Barcelona Metropolitan Area. In: Zagreb, $41^{\text {st }}$ Congress of the European Regional Science Association, agosto 2001.

MCMILLEN, D. \& MCDONALD, J. A nonparametric analysis of employment density in a polycentric city. Journal of Regional Science, 1997, vol. 37, p.591-612.

MIRALLES, C. Ciudad y transporte un binomio imperfecto. Barcelona: Ariel Geografía, 2002. Disponible en Internet: http:// www3.interscience.wiley.com/cgi-bin/fulltext/118779126/PDFSTART

MIRALleS, C. y CeBOlladA, Á. Movilidad y transporte. Opciones políticas para la ciudad. Barcelona: Laboratorio de Alternativas Barcelona, documento de trabajo 25, 2003. Disponible en Internet: http://www. falternativas.org/laboratorio/documentos/ documentos-de-trabajo/(offset)/130

NEL·LO, O. Cataluña, ciudad de ciudades. Lleida: Milenio, 2002.
ORTIZ, J. y MORALES, S. Impacto socioespacial de las migraciones intraurbanas en entidades de centro y de nuevas periferias del Gran Santiago. EURE, 2002, vol. 28, $N^{\circ}$ 85, p. 171-185. Disponible en Internet: http://redalyc.uaemex.mx/redalyc/src/inicio/ ArtPdfRed.jsp? $i$ Cve $=19608509 \& i C$ veNum $=0$

OYON, J. Transporte público y estructura urbana: (de mediados s. XIX a mediados s. XX): Gran Bretaña, Francia y países germánicos. Ecología Política, 1999, vol. 17, p. 17-35.

ROCA, J.; MARMOLEJO, C. \& MOIX, M. Urban structure and polycentrism: towards a redefinition of the sub-centre concept. Urban Studies, 2009, vol. 46, No 13, p. 2841-2868.

RODRÍGUEZ, I. Vivienda social latinoamericana: la clonación del paisaje de la exclusión. $A C E, 2006$, vol. 1, No 2. Disponible en Internet: http://www-cpsv.upc.es/ACE/ Articles-n2/seccion-articulos/TEM_isabelrodriguez_ARTICULO.pdf

RODRÍGUEZ-BACHILLER, A. Discontiguous urban growth and the new urban economics: a review. Urban Studies, 1986, vol. 23, $\mathrm{N}^{\circ} 2$, p.79-104.

THUILLIER, G. El impacto socioespacial de las urbanizaciones cerradas: El caso de la Región Metropolitana de Buenos Aires. EURE, 2005, vol. 31, No 93, p. 5-20. Disponible en Internet: http://redalyc. uaemex.mx/redalyc/src/inicio/ArtPdfRed. jsp? $\mathrm{iCve}=19609301 \& \mathrm{iCveNum}=0$

VAN DER BERG, L. Urban Europe: a study of growth and decline. Ciudad: Pergamon Press, 1982.

VAN WEE, B.; RIETVELD, P. \& MEURS, H. Is average daily travel time expenditure constant? In search of explanations for an increase in average travel time. Journal of transport geography, 2006, vol. 14, p. 109-122.

VIDAL-KOPPMANN, S. Mutaciones metropolitanas: de la construcción de barrios cerrados a la creación de ciudades privadas: balance de una década de urbanización privada en la Región Metropolitana de Buenos Aires. En: Barcelona, X Coloquio Internacional de Geocrítica, mayo 2008. 